\title{
Robotergestützte nicht besser als konventionelle Chirurgie
}

— Prof. Axel Merseburger, Hannover, hat Leitlinienempfehlungen $\mathrm{zu}$ roboterassistierten urologischen Operationen vorgestellt. Er präsentierte Daten einer Metaanalyse mit 46 Studien zur robotergestützten Teilnephrektomie (RAPN) versus laparoskopische Teilnephrektomie (LPN) sowie zur offenen radikalen Prostatektomie (ORP) versus robotergestützte radikale Prostatektomie (RARP) versus laparoskopische radikale Prostatektomie (LRP).

Die Roboter-assistierten Methoden hätten zwar Vorteile gegenüber den etablierten, es sei jedoch unklar, ob diese Vorteile klinisch relevant sind, erklärte Merseburger. So ist die RAPN zwar schneller durchzuführen als die LPN und mit einer kürzeren Krankenhausverweil- dauer assoziiert. Allerdings liegen keine verlässlichen Daten zum onkologischen Outcome und den Komplikationsraten vor. RARP ist der ORP in punkto Krankenhausverweildauer und geringerem Blutverlust überlegen. Dafür dauert die Operation länger und Daten zu Komplikationsraten und Erhalt der erektilen Funktion sind widersprüchlich. Alles in allem lägen die Evidenzlevel jeweils nur bei 3 oder 4 .

„Solange keine randomisierten klinischen Vergleichsstudien vorliegen, ist es unmöglich, den Vorteil der einen Methode gegenüber anderen Techniken zu ermitteln“, betonte Merseburger. So könne man beispielsweise nicht mit Sicherheit sagen, ob die Operateure gleich gut ausgebildet und erfahren sind, wenn man die derzeitigen Studien vergleicht. Eine Lösung wäre, spezialisierte Zentren mit validierten Trainingsprogrammen für beide Operationstechniken einzuführen. EAU-Generalsekretär Prof. Per-Anders Abrahamsson ging sogar noch einen Schritt weiter: „Wenn wir in ein Flugzeug steigen, setzen wir voraus, dass der Pilot gut ausgebildet, geprüft und auf dem aktuellsten Stand ist. Auch Operateure müssen eine Lizenz zum Operieren haben, vor allem wenn ein hochkomplizierter Operationsroboter involviert ist“. Solange die Erfahrung des Operateurs eine dominierende Rolle spielt, bleibt die Wahl der Methode weiterhin zweitrangig.

slx

P 187, P 257, EAU-Pressekonferenz

\section{Urologen sind Leitlinien-Muffel}

—Leitlinien dienen dem Arzt als Orientierungshilfe für die Behandlung einer Krankheit. Dass Theorie und Wirklichkeit nicht immer zusammenpassen, zeigen zwei aktuelle Beispiele aus der Diagnostik und Therapie des Prostatakarzinoms sowie dem Lymphknotenmanagement beim Peniskarzinom.

Laut EAU-Leitlinien (www.uroweb.org/ guidelines) ist beim fortgeschrittenen Prostatakarzinom (PCa) die Androgendeprivationstherapie (ADT) indiziert. Doch die Compliance der behandelnden Urologen ist laut Prof. Axel Heidenreich, Aachen, eher gering. Das belegen retrospektive Daten von 2.778 Patienten. Unter anderem war bei $12,2 \%$ der Patienten unklar, ob eine systemische Metastasierung vorliegt und in 60,9\% der Fälle der Lymphknotenstatus unbekannt. Zudem wurde nur bei $21,5 \%$ der Patienten Testosteron bestimmt und bei kaum einem der
Patienten wurden im Follow-up die kardiovaskulären Effekte einer Langzeit-ADT überwacht.

Etwas besser ist die Compliance der Urologen in der Diagnose des PCa. Dennoch schicken nur $66 \%$ der Urologen Biopsieproben in separaten Gefäßen zur histologischen Untersuchung. Bemängelt wurden auch ein zu frühes oder unnötiges Anwenden von bildgebenden Verfahren: So wurden $24 \%$ der Niedrig-Risiko-Patienten einer Abdomen-Computertomografie unterzogen, $18 \%$ einer Skelettszintigrafie. Zudem wurden die Verfahren Magnetresonanztomografie (12\%) und Positronen-Emissions-Tomografie (6,5\%) eingesetzt, die beide nicht empfohlen werden. Eine bessere Verbreitung und Compliance der Leitlinien sei dringend erforderlich, so Heidenreich.

Auch im Falle des Peniskarzinoms gehen Leitlinienempfehlungen und kli- nische Praxis weit auseinander. Das verdeutlicht die Auswertung eines neun Fragen zum Lymphknotenmanagement beim Peniskarzinom umfassenden Fragebogens der Uni Rostock, der an alle 280 urologischen Abteilungen in Deutschland versandt wurde. Nur $57 \%$ der 129 urologischen Abteilungen, die an der Umfrage teilnahmen, wendeten die in den EAURichtlinien empfohlene Risiko-Klassifikation an. Zudem variierte die Zahl der Patienten, bei denen eine inguinale Lymphadenektomie (LAD) durchgeführt wurde, extrem (0-100\%). In $68 \%$ der urologischen Abteilungen wurden zumindest $50 \%$ der Patienten einer LAD unterzogen. Auch die Rostocker Kollegen plädieren für ein einheitliches Vorgehen entsprechend der Leitlinien.

slx

P 302, P 1093, P 1103 